\title{
Errata to
}

"The Dirichlet series of $\zeta(s) \zeta^{\alpha}(s+1) f(s+1)$ :

\section{On an error term associated with its coefficients"}

\author{
(Acta Arith. 75 (1996), 39-69)
}

by

U. Balakrishnan (Singapore) and Y.-F. S. PÉtermann (Genève)

1. Hypotheses in Theorem 2. We recall the statement of our Theorem 2 in Section 3 (page 50).

THEOREM 2. Let $v_{n}=v(n)$ be a real multiplicative arithmetical function satisfying, for some real numbers $\alpha>0$ and $\beta \geq 0$,

$$
\begin{gathered}
\sum_{n \leq x}\left|v_{n}\right|=O\left((\log x)^{\alpha}\right) ; \\
\sum_{n \leq x}\left(n v_{n}\right)^{2}=O\left(x(\log x)^{\beta}\right) ;
\end{gathered}
$$

(h3) $p^{k} v\left(p^{k}\right)$ is an ultimately monotonic function of $p$ for $k=1$ and $k=2$, and is bounded for every $k \geq 1$.

Then, if we set $y:=x \exp \left(-(\log x)^{b}\right)$ for some positive number $b, t:=\log x$, and $u:=\log t=\log \log x$, we have

$$
\sum_{n \leq y} v_{n} \psi(x / n)=O\left(t^{2 \alpha / 3} u^{4 \alpha / 3}\right) .
$$

Hypothesis (h1) is insufficient to ensure the validity of the proof sketched in the paper (the last argument in the proof of Lemma 3.3 on pages 52-53 is not valid under that too weak hypothesis). It can however be replaced by

$$
\sum_{n \leq x} n\left|v_{n}\right|=x \sum_{r=0}^{\lambda+[\alpha]} F_{r}(\log x)^{\alpha-r}+O\left(x(\log x)^{-\lambda}\right)
$$

for every positive natural number $\lambda$ (and where the $F_{r}$ are real constants). A complete proof of Theorem 2 under hypothesis (h1*) (and under a weakened hypothesis $(\mathrm{h} 3 *))$ is given in [2]. And as noted in the latter (Section 4), the 
stronger hypothesis $(\mathrm{h} 1 *)$ is still satisfied by all the examples we treat in our paper, and in particular by $a(n)=(1 * v)(n)=(\sigma(n) / n)^{r}$ or $(\phi(n) / n)^{r}$ or $(\sigma(n) / \phi(n))^{r}$.

2. A mistake in Saltykov's paper. For the second time, we thank Professor A. Schinzel for twisting our arm to make us read [3]. The first time we did so was in the note "added in proof" of our paper, in which we claimed that A. I. Saltykov's result

$$
H(x)=O\left((\log x)^{2 / 3}(\log \log x)^{1+\varepsilon}\right)
$$

for the error term

$$
H(x)=\sum_{n \leq x} \phi(n) / n-6 x / \pi^{2},
$$

which is a better estimate than Walfisz'

$$
H(x)=O\left((\log x)^{2 / 3}(\log \log x)^{4 / 3}\right)
$$

(proved in [4]), is correct. We then realised that the reason why Saltykov's paper was considered suspect, is because it makes use of a theorem proved in [1], in which Korobov also makes an unproved claim on the zeros of the Riemann zeta function. (Korobov's paper, together with a paper of Vinogradov, in which the same unverified claim is made, is the starting point of a famous controversy which was never really completely settled.) But we also realised that the theorem Saltykov uses is perfectly sound, and we concluded in our note that his estimate on $H(x)$ was undisputable and the best to date. We even announced an improvement of our Theorem 2 based on Saltykov's ideas.

Our reading of [3] was however not careful enough (was Professor Schinzel's twist not strong enough?), and Pétermann [2] discovered later a mistake in Saltykov's argument. More exactly, the proof of his Lemma 2.6, which states an estimate involving two parameters $\gamma_{1}$ and $\gamma_{2}$, is not valid for $\gamma_{1}=0$. This value $\gamma_{1}=0$, however, is the value needed in order to deduce (1) from Saltykov's result. And it is clear that the modification needed in Lemma 2.6 in order to treat the case $\gamma_{1}=0$ "only" yields Walfisz' estimate (2), which thus cannot be improved with the method. More details on this can be found in $[2]$ (Section 5).

\section{References}

[1] M. N. Korobov, Estimates of trigonometrical sums and their applications, Uspekhi Mat. Nauk 13 (1958), no. 4, 185-192 (in Russian).

[2] Y.-F. S. Pétermann, On an estimate of Walfisz and Saltykov for an error term related to the Euler function, J. Théor. Nombres Bordeaux, to appear. 
[3] A. I. Saltykov, On Euler's function, Vestnik Moskov. Univ. Ser. I Mat. Mekh. 1960, no. 6, 34-50 (in Russian).

[4] A. Walfisz, Weylsche Exponentialsummen in der neueren Zahlentheorie, Deutscher Verlag Wiss., Berlin, 1963.

19, Jalan Gembira

Singapore 369125
Section de Mathématiques

Université de Genève

2-4, rue du Lièvre, C.P. 240

1211 Genève 24, Suisse

E-mail: peterman@ibm.unige.ch 